\title{
Lepton-flavor-violating Higgs decay $h \rightarrow \mu \tau$ and muon anomalous magnetic moment in a general two Higgs double model
}

\author{
Kazuhiro Tobe*t \\ Department of Physics and Kobayashi-Maskawa Institute for the Origin of Particles and the \\ Universe, Nagoya University \\ E-mail: tobedeken.phys.nagoya-u.ac.jp
}

\begin{abstract}
In a general two Higgs double model, flavor-changing phenomena mediated by neutral Higgs bosons are predicted. We find the $\mu-\tau$ flavor-violating Higgs interactions in a general Higgs doublet model can explain the excess event in a Higgs decay $h \rightarrow \mu \tau$ observed by the CMS collaboration as well as the anomaly of the muon anomalous magnetic moment, simultaneously. We also discuss that the predicition of $\tau \rightarrow \mu \gamma$ in the scenario can be within the reach of the future B-factory.
\end{abstract}




\section{Introduction}

Since the standard model (SM) well-describes phenomena up to the electroweak scale, it is considered to be a very successful theory for elementary particles. The Higgs boson discovery further strengthens the success of the SM. On the other hand, there are a few phenomena which are not explained by the current understanding of the SM. One of them is the anomalous magnetic moment of muon (muon g-2). A discrepancy between the measured value $\left(a_{\mu}^{\text {Exp }}\right)$ and the SM prediction $\left(a_{\mu}^{\mathrm{SM}}\right)$ of the muon $\mathrm{g}-2$ has been reported [2]. For example, see [3], $\delta a_{\mu}=a_{\mu}^{\mathrm{Exp}}-a_{\mu}^{\mathrm{SM}}=$ $(26.1 \pm 8.0) \times 10^{-10}$, and so far no clear understanding of this anomaly has been established. Another one is the event excess of the Higgs boson decay $h \rightarrow \mu \tau$, reported by the CMS collaboration at the Large Hadron Collider (LHC) experiment [4], $\mathrm{BR}(h \rightarrow \mu \tau)=\left(0.84_{-0.37}^{+0.39}\right) \%$, which never occurs in the SM. These phenomena would be good hints for the physics beyond the SM. In this presentation, we show that these two anomalies in the muon g- 2 and $h \rightarrow \mu \tau$ can be explained by the $\mu-\tau$ flavor-violating interactions in a general two Higgs doublet model (2HDM), and we also discuss the prediction of the scenario.

\section{General two Higgs doublet model}

In a general two Higgs doublet model (2HDM), both Higgs doublets can couple to all fermions and hence the Yukawa interactions are given by

$$
\mathscr{L}=-\bar{L}_{L}^{i} H_{1} y_{e}^{i} e_{R}^{i}-\bar{L}_{L}^{i} H_{2} \rho_{e}^{i j} e_{R}^{j}+\text { h.c. }
$$

where $L_{L}=\left(V_{\mathrm{MNS}} v_{L}, e_{L}\right)^{\mathrm{T}}$, where $V_{\mathrm{MNS}}$ is the Maki-Nakagawa-Sakata matrix. Here we take a basis where only Higgs doublet $H_{1}$ gets a vacuum expectation value (vev),

$$
H_{1}=\left(\begin{array}{c}
G^{+} \\
\frac{v+\phi_{1}+i G}{\sqrt{2}}
\end{array}\right), H_{2}=\left(\begin{array}{c}
H^{+} \\
\frac{\phi_{2}+i A}{\sqrt{2}}
\end{array}\right),\left(\begin{array}{c}
\phi_{1} \\
\phi_{2}
\end{array}\right)=\left(\begin{array}{cc}
\cos \theta_{\beta \alpha} & \sin \theta_{\beta \alpha} \\
-\sin \theta_{\beta \alpha} & \cos \theta_{\beta \alpha}
\end{array}\right)\left(\begin{array}{c}
H \\
h
\end{array}\right),
$$

where $G^{+}$and $G$ are Nambu-Goldstone bosons and $H^{ \pm}$and $A$ are charged and CP-odd Higgs bosons, respectively. Thus the Yukawa interactions shown above are written in the fermion mass eigenstates. ${ }^{1}$ The CP-even neutral Higgs bosons $\phi_{1,2}$ can mix and form the mass eigenstates $h$ and $H$ where $h$ is a SM-like Higgs boson. For small $\left|c_{\beta \alpha}\right|\left(c_{\beta \alpha}=\cos \theta_{\beta \alpha}\right), \phi_{1} \simeq h$. Note that the Yukawa couplings $\rho_{e}$ are flavor-violating interactions in general. The mass spectrum of neutral Higgs bosons in this model are expressed as

$$
m_{H^{+}}^{2}=m_{A}^{2}+\frac{\lambda_{5}-\lambda_{4}}{2} v^{2}, m_{H}^{2} \simeq m_{A}^{2}+\lambda_{5} v^{2}
$$

where $\lambda_{4,5}$ are Higgs quartic couplings $\left(H_{1}^{\dagger} H_{2}\right)\left(H_{2}^{\dagger} H_{1}\right)$ and $\left(H_{1}^{\dagger} H_{2}\right)^{2} / 2$, respectively, in the treelevel Higgs potential.

\footnotetext{
${ }^{1}$ The Yukawa couplings in quark sector can also be written similarly.
} 

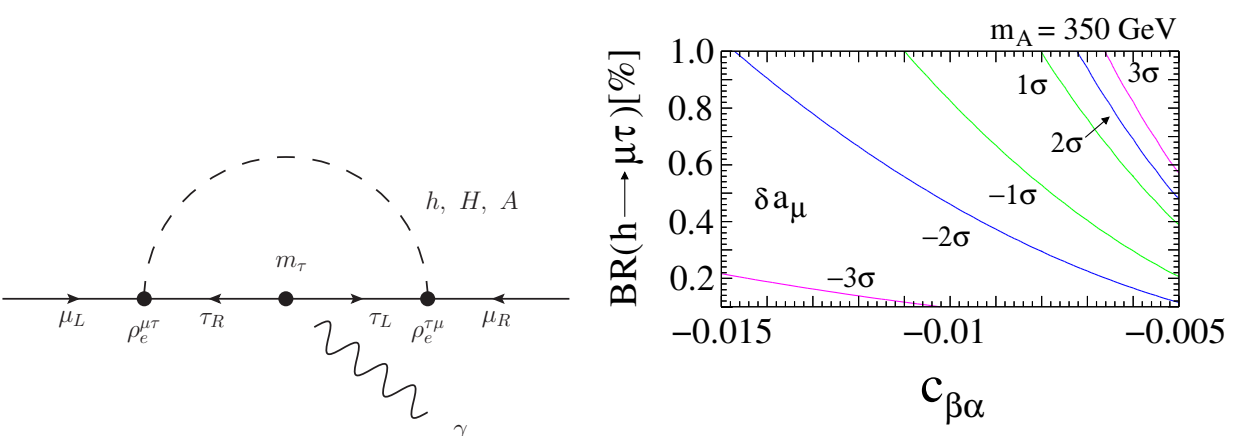

Figure 1: (Left) A Feynman diagram for neutral Higgs boson contributions to the muon g-2 via the $\mu-\tau$ flavor-violating Yukawa couplings $\rho_{e}^{\mu \tau(\tau \mu)}$. A photon is attached somewhere in the charged lepton line. (Right) Neutral Higgs contributions to the muon $\mathrm{g}-2\left(\delta_{\mu}\right)$ as a function of $\operatorname{BR}(h \rightarrow \mu \tau)[\%]$ and $c_{\beta \alpha}(=$ $\left.\cos \theta_{\beta \alpha}\right)$. The lines where the muon g-2 anomaly is explained within $\pm 1 \sigma, \pm 2 \sigma$ and $\pm 3 \sigma$ are shown. Here we have assumed that $m_{A}=350 \mathrm{GeV}, \lambda_{4}=\lambda_{5}=1$ and $\rho_{e}^{\tau \mu}=-\rho_{e}^{\mu \tau}$.

\section{3. $h \rightarrow \mu \tau$ and muon g-2}

The flavor-violating Yukawa couplings $\rho_{e}^{\mu \tau(\tau \mu)}$ can easily explain the CMS event excess in $h \rightarrow \mu \tau$ if the size of the Yukawa couplings satisfies the following condition,

$$
\bar{\rho}^{\mu \tau} \equiv \sqrt{\frac{\left|\rho_{e}^{\mu \tau}\right|^{2}+\left|\rho_{e}^{\tau \mu}\right|^{2}}{2}} \simeq 0.26\left(\frac{0.01}{\left|c_{\beta \alpha}\right|}\right) \sqrt{\frac{\mathrm{BR}(h \rightarrow \mu \tau)}{0.84 \times 10^{-2}}} .
$$

These $\mu-\tau$ flavor-violating Yukawa couplings $\rho_{e}^{\mu \tau(\tau \mu)}$ can induce extra contributions to the muon g-2, mediated by neutral Higgs bosons, as shown in Fig. 1 (Left). Note that the extra-contributions to the muon g-2 are proportional to the $\tau$ mass, which is necessary to flip the chirality of the muon and induces the $O\left(m_{\tau} / m_{\mu}\right)$ enhancement in the muon g-2, compared to one induced by the flavor diagonal Yukawa coupling. Therefore, the $\mu-\tau$ flavor violation is essential to have the large extra-contributions to the muon g-2. Assuming that $m_{A}=350 \mathrm{GeV}, \lambda_{4}=\lambda_{5}=1$ and $\rho_{e}^{\tau \mu}=-\rho_{e}^{\mu \tau}$, the numerical values of extra contributions to muon $\mathrm{g}-2\left(\delta a_{\mu}\right)$ are shown as a function of $\mathrm{BR}(h \rightarrow \mu \tau)[\%]$ and $c_{\beta \alpha}\left(=\cos \theta_{\beta \alpha}\right)$ in Fig. 1(Right). The lines where the muon g-2 anomaly is explained within $\pm 1 \sigma, \pm 2 \sigma$ and $\pm 3 \sigma$ are displayed. One can see that the both anomalies in $h \rightarrow \mu \tau$ and the muon g- 2 can be explained by the $\mu-\tau$ flavor-violating interactions in the general $2 \mathrm{HDM}$ when $\left|c_{\beta \alpha}\right|$ is small.

\section{4. $\tau \rightarrow \mu \gamma$}

The large $\mu-\tau$ flavor violation also generates $\tau \rightarrow \mu \gamma$. Feynman diagrams of the dominant one-loop and (Barr-Zee type) two-loop contributions are depicted in Fig. 2. It is known that the Barr-Zee type two-loop contribution can be dominant because of the large top Yukawa coupling [5]. We find that even if unknown Yukawa couplings $\rho_{f}(f=e, u, d)$ other than $\rho_{e}^{\mu \tau(\tau \mu)}$ are negligibly small, the predicted branching ratio of $\tau \rightarrow \mu \gamma$ can be as large as $10^{-9}$, which might be within the reach of the future B-factory. If extra-Yukawa couplings, such as $\rho_{u}^{t t}$ and $\rho_{e}^{\tau \tau}$, are non-zero, 

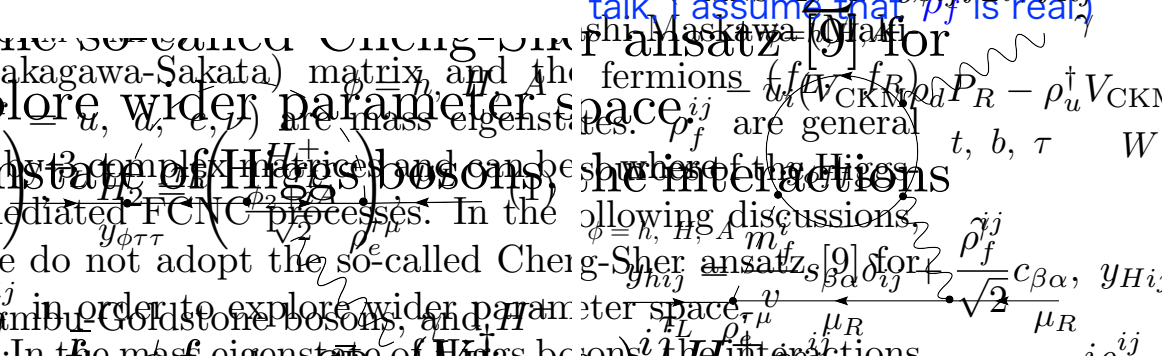

Figure 2: Feynman diagrams for the dominant one-loop and (Barr-Zee type) two-loop contributions to $\tau \rightarrow \mu \gamma$.

they can enhance the rate of $\tau \rightarrow \mu \gamma$. In Fig. 3, the branching ratio of $\tau \rightarrow \mu \gamma$ is shown as a function of $\rho_{e}^{\tau \tau}$ and $\rho_{u}^{t t}$. Here, we have assumed that $m_{A}=350 \mathrm{GeV}, \lambda_{4}=\lambda_{5}=1, c_{\beta \alpha}=-0.008$ and $\operatorname{BR}(h \rightarrow \mu \tau)=0.84 \%$ with $\rho_{e}^{\tau \mu}=-\rho_{e}^{\mu \tau}$. For this parameter set, $\delta a_{\mu}$ is $2.9 \times 10^{-9}$, which explains the muon g-2 anomaly within $1 \sigma$. In Fig. 3, lines of $\operatorname{BR}(\tau \rightarrow \mu \gamma)$ for $4.4 \times 10^{-8}$ (current experimental limit) and $10^{-9}$ are shown. As one can see from Fig. 3, the future improvement of the branching ratio at the $10^{-9}$ level would have the great impact on proving this scenario. For further detail of the phenomenological analysis for this model, see Ref. [6].

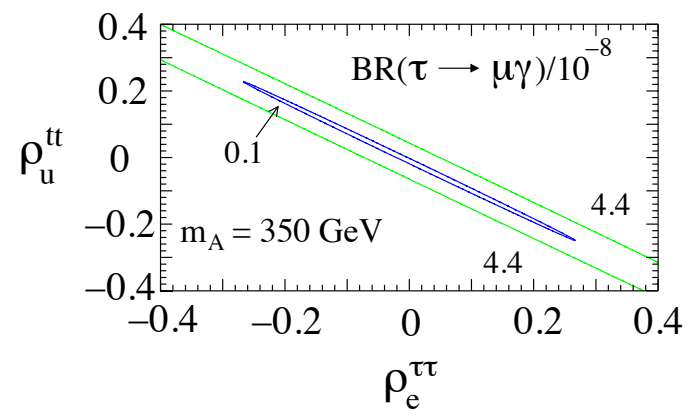

Figure 3: Branching ratio of $\tau \rightarrow \mu \gamma$ is shown as a function of $\rho_{e}^{\tau \tau}$ and $\rho_{u}^{t t}$. Here lines of $\operatorname{BR}(\tau \rightarrow \mu \gamma)$ for $4.4 \times 10^{-8}$ (current limit) and $10^{-9}$ are depicted. We have assumed that $m_{A}=350 \mathrm{GeV}$ and $\lambda_{4}=\lambda_{5}=1$, $c_{\beta \alpha}=-0.008$ and $\operatorname{BR}(h \rightarrow \mu \tau)=0.84 \%$ with $\rho_{e}^{\tau \mu}=-\rho_{e}^{\mu \tau}$. We note that for this parameter set, $\delta a_{\mu}=$ $2.9 \times 10^{-9}$, which can explain the muon g-2 anomaly within $1 \sigma$.

\section{References}

[1] Y. Omura, E. Senaha and K. Tobe, JHEP 1505 (2015) 028 [arXiv:1502.07824 [hep-ph]].

[2] K. A. Olive et al. [Particle Data Group Collaboration], Chin. Phys. C 38 (2014) 090001.

[3] K. Hagiwara et al., J. Phys. G 38 (2011) 085003 [arXiv:1105.3149 [hep-ph]].

[4] V. Khachatryan et al. [CMS Collaboration], arXiv:1502.07400 [hep-ex].

[5] D. Chang, W. S. Hou and W. Y. Keung, Phys. Rev. D 48, 217 (1993) [hep-ph/9302267].

[6] Y. Omura, E. Senaha and K. Tobe, work in progress. 